\title{
On inequalities of Jensen-Ostrowski type
}

Pietro Cerone', Sever S Dragomir ${ }^{2,3}$ and Eder Kikianty ${ }^{4 *}$

"Correspondence: eder.kikianty@gmail.com

${ }^{4}$ Department of Pure and Applied Mathematics, University of Johannesburg, P.O. Box 524, Auckland Park, 2006, South Africa Full list of author information is available at the end of the article

\begin{abstract}
We provide new inequalities of Jensen-Ostrowski type, by considering bounds for the magnitude of $\int_{\Omega} f \circ g d \mu-f(\zeta)-\left(\int_{\Omega} g d \mu-\zeta\right) f^{\prime}(\zeta)-\frac{1}{2} \lambda \int_{\Omega}(g-\zeta)^{2} d \mu, \zeta \in[a, b]$, with various assumptions on the absolutely continuous function $f:[a, b] \rightarrow \mathbb{C}$ and $a$ $\mu$-measurable function $g$, and a complex number $\lambda$. Inequalities of Ostrowski and Jensen type are obtained as special cases, by setting $\lambda=0$ and $\zeta=\int_{\Omega} g d \mu$, respectively. In particular, we obtain some bounds for the discrepancy in Jensen's integral inequality. Applications of these inequalities for $f$-divergence measures are also given.
\end{abstract}

MSC: Primary 26D10; 26D15; secondary 94A17

Keywords: Jensen inequality; Ostrowski inequality; divergence measure; discrepancy

\section{Introduction}

The simplest form of Jensen's inequality for a convex function $f: I \rightarrow \mathbb{R}$ reads as follows:

$$
f\left(\frac{a+b}{2}\right) \leq \frac{f(a)+f(b)}{2}, \quad a, b \in I .
$$

This was proved by Jensen in 1906 [1]. Throughout the paper, $\mathbb{R}$ and $\mathbb{C}$ denote the set of real numbers and the set of complex numbers, respectively. Let $(\Omega, \mathcal{A}, \mu)$ be a measurable space with $\int_{\Omega} d \mu=1$, consisting of a set $\Omega$, a $\sigma$-algebra $\mathcal{A}$ of subsets of $\Omega$ and a countably additive and positive measure $\mu$ on $\mathcal{A}$ with values in the set of extended real numbers. Jensen's (integral) inequality now takes the following form: for a $\mu$-integrable function $g: \Omega \rightarrow[m, M] \subset \mathbb{R}$ and a convex function $f:[m, M] \rightarrow \mathbb{R}$, we have

$$
f\left(\int_{\Omega} g d \mu\right) \leq \int_{\Omega} f \circ g d \mu .
$$

Costarelli and Spigler [2] considered the sharpness of Jensen's integral inequality (for real-valued convex function $f$ and nonnegative function $g$ ) by studying bounds for the discrepancy in the inequality.

Proposition 1 (Costarelli and Spigler [2]) Let $\varphi: I \rightarrow \mathbb{R}$ be a real-valued function, where $I$ is a connected bounded set in $\mathbb{R}$, and $f:[0,1] \rightarrow I$ a real-valued nonnegative function where $f \in L^{1}(0,1)$. If $\varphi$ is a $C^{2}$-function, then

$$
\varphi(f(x))=\varphi(c)+\varphi^{\prime}(c)[f(x)-c]+\frac{1}{2} \varphi^{\prime \prime}\left(c^{*}(x)\right)[f(x)-c]^{2}, \quad x \in[0,1],
$$

(c) 2015 Cerone et al. This article is distributed under the terms of the Creative Commons Attribution 4.0 International License (http://creativecommons.org/licenses/by/4.0/), which permits unrestricted use, distribution, and reproduction in any medium, provided you give appropriate credit to the original author(s) and the source, provide a link to the Creative Commons license, and indicate if changes were made. 
where $c=f\left(x_{0}\right)$, which can be chosen arbitrarily in the domain of $\varphi$ such that $f\left(x_{0}\right) \in \stackrel{\circ}{I}$ (here, $\stackrel{I}{I}$ is the interior of $I)$, and $c^{*}(x)$ is a suitable value between $f(x)$ and $f\left(x_{0}\right)$. Furthermore,

$$
\int_{0}^{1} \varphi(f(x)) d x=\varphi(c)+\varphi^{\prime}(c) \int_{0}^{1}[f(x)-c] d x+\frac{1}{2} \int_{0}^{1} \varphi^{\prime \prime}\left(c^{*}(x)\right)[f(x)-c]^{2} d x .
$$

If $\varphi$ is convex and $f$ is nonnegative, then the discrepancy in the Jensen inequality is given by the following estimates:

$$
\begin{aligned}
0 & \leq \int_{0}^{1} \varphi(f(x)) d x-\varphi\left(\int_{0}^{1} f(x) d x\right) \\
& \leq \frac{1}{2}\left\|\varphi^{\prime \prime}\right\|_{L^{\infty\left(I_{2}\right)}}\left[\|f-c\|_{L^{2}}^{2}+\|f-c\|_{L^{1}}^{2}\right],
\end{aligned}
$$

where $I_{2}$ denotes the domain of $\varphi^{\prime \prime}$. Furthermore, if $\varphi$ is a $C^{2}$-smooth function, then we have

$$
\begin{aligned}
0 & \leq \int_{0}^{1} \varphi(f(x)) d x-\varphi\left(\int_{0}^{1} f(x) d x\right) \\
& \leq \frac{1}{2}\left\|\varphi^{\prime \prime}\right\|_{L^{\infty}\left(I^{2}\right)}\|f-c\|_{L^{2}}^{2}-\frac{1}{2} \inf _{I_{2}} \varphi^{\prime \prime}\left[\int_{0}^{1}(f(x)-c) d x\right]^{2} .
\end{aligned}
$$

Further inequalities involving bounds for the discrepancy in Jensen type inequalities for general integrals are given in [3] and [4].

In 1938, Ostrowski proved the following inequality [5].

Proposition 2 Let $f:[a, b] \rightarrow \mathbb{R}$ be continuous on $[a, b]$ and differentiable on $(a, b)$ such that $f^{\prime}:(a, b) \rightarrow \mathbb{R}$ is bounded on $(a, b)$, i.e., $\left\|f^{\prime}\right\|_{\infty}:=\sup _{t \in(a, b)}\left|f^{\prime}(t)\right|<\infty$. Then

$$
\left|f(x)-\frac{1}{b-a} \int_{a}^{b} f(t) d t\right| \leq\left[\frac{1}{4}+\left(\frac{x-\frac{a+b}{2}}{b-a}\right)^{2}\right]\left\|f^{\prime}\right\|_{\infty}(b-a)
$$

for all $x \in[a, b]$ and the constant $\frac{1}{4}$ is the best possible.

In what follows, we recall a generalisation of Ostrowski's inequality for twice differentiable mappings.

Proposition 3 (Cerone et al. [6]) Let $f:[a, b] \rightarrow \mathbb{R}$ be a mapping such that the derivative $f^{\prime}:[a, b] \rightarrow \mathbb{R}$ is absolutely continuous on $[a, b]$. Then we have the inequality

$$
\begin{aligned}
& \left|\int_{a}^{b} f(t) d t-(b-a) f(x)+(b-a)\left(x-\frac{a+b}{2}\right) f^{\prime}(x)\right| \\
& \quad \leq\left[\frac{1}{24}+\frac{1}{2} \frac{\left(x-\frac{a+b}{2}\right)^{2}}{(b-a)^{2}}\right](b-a)^{3}\left\|f^{\prime \prime}\right\|_{\infty}
\end{aligned}
$$

for all $x \in[a, b]$.

We refer the readers to the book by Mitrinović et al. [7] and the book by Dragomir and Rassias [8] for further generalisations of Ostrowski's inequality.

Dragomir [9] introduced some inequalities which combine the two aforementioned inequalities, referred to as the Jensen-Ostrowski type inequalities. We recall one of the results in the next proposition. 
Proposition 4 Let $\Phi: I \rightarrow \mathbb{C}$ be an absolutely continuous functions on $[a, b] \in \stackrel{\circ}{I}$, the interior of I. If $g: \Omega \rightarrow[a, b]$ is Lebesgue $\mu$-measurable on $\Omega$ and $\Phi \circ g, g \in L(\Omega, \mu)$, then

$$
\begin{aligned}
& \left|\int_{\Omega} \Phi \circ g d \mu-\Phi(x)-\lambda\left(\int_{\Omega} g d \mu-x\right)\right| \\
& \quad \leq \int_{\Omega}|g-x|\left\|\Phi^{\prime}((1-\ell) x+\ell g)-\lambda\right\|_{[0,1], 1} d \mu \\
& \quad \leq\left\{\begin{array}{l}
\|g-x\|_{\Omega, \infty}\|\| \Phi^{\prime}((1-\ell) x+\ell g)-\lambda\left\|_{[0,1], 1}\right\|_{\Omega, 1}, \\
\|g-x\|_{\Omega, p}\|\| \Phi^{\prime}((1-\ell) x+\ell g)-\lambda\left\|_{[0,1], 1}\right\|_{\Omega, q}, \quad p>1, \frac{1}{p}+\frac{1}{q}=1 \\
\|g-x\|_{\Omega, 1}\|\| \Phi^{\prime}((1-\ell) x+\ell g)-\lambda\left\|_{[0,1], 1}\right\|_{\Omega, \infty},
\end{array}\right.
\end{aligned}
$$

for any $\lambda \in \mathbb{C}$ and $x \in[a, b]$. Here, $\ell$ denotes the identity function on $[0,1]$, namely $\ell(t)=t$, for $t \in[0,1]$.

Inequalities of Jensen type and Ostrowski type are obtained by setting $x=\int_{\Omega} g d \mu$ and $\lambda=0$, respectively, in Proposition 4. Further results on inequalities for functions with bounded derivatives and applications for $f$-divergence measures in information theory are also given in [9]. Similar inequalities are given for: (i) functions with derivatives that are of bounded variation and Lipschitz continuous in [10]; and (ii) functions which absolute values of the derivatives are convex in [11]. In [12], new inequalities of Jensen-Ostrowski type are established by obtaining bounds for the magnitude of

$$
\int_{\Omega}(f \circ g) d \mu-f(\zeta)-\int_{\Omega}(g-\zeta) f^{\prime} \circ g d \mu+\frac{1}{2} \lambda \int_{\Omega}(g-\zeta)^{2} d \mu, \quad \zeta \in[a, b]
$$

for various assumptions on the absolutely continuous function $f:[a, b] \rightarrow \mathbb{C}$, a $\mu$-measurable function $g$ and $\lambda \in \mathbb{C}$.

In this paper, we provide new inequalities of Jensen-Ostrowski type by studying the magnitude of:

$$
\int_{\Omega} f \circ g d \mu-f(\zeta)-\left(\int_{\Omega} g d \mu-\zeta\right) f^{\prime}(\zeta)-\frac{1}{2} \lambda \int_{\Omega}(g-\zeta)^{2} d \mu, \quad \zeta \in[a, b],
$$

following our previous results in [12]. Our results in this paper stem on the estimate obtained by utilising the Taylor approximation with integral remainders ( $c f$. Lemma 1 of Section 2).

We present our main results in Section 3. We obtain inequalities with bounds involving the $p$-norms $(1 \leq p \leq \infty)$, as well as inequalities for functions with bounded and convex second derivatives. Applications for $f$-divergence measure are provided in Section 5 .

In Section 4, we discuss some special cases of our results. We provide a generalised version of the Ostrowski inequality (1.8) (cf. Proposition 3) in the measure-theoretic (and probabilistic) form in Remark 3. We also obtain a result on the discrepancy in Jensen's inequality ( $c f$. inequality (4.2)), without the assumption of convexity. We connect this result with those of Costarelli and Spigler [2] (cf. Proposition 1) in Remark 4. Costarelli and Sprigler noted that the bound in (1.6) is better than (1.5) due to a stronger assumption of $C^{2}$-smoothness. Under the assumptions of Proposition 1, our result gives a better upper bound than (1.5), although (1.6) still gives the better upper bound. However, our result holds in a more general setting, that is, for differentiable functions with absolutely continuous derivatives, in a measure-theoretic (and probabilistic) form. 


\section{Some estimates}

We start with the following lemma to assist us in our calculations throughout the paper.

Lemma 1 Let $f: I \rightarrow \mathbb{C}$ be a differentiable function on $\stackrel{\circ}{I}, f^{\prime}:[a, b] \subset \stackrel{\circ}{I} \rightarrow \mathbb{C}$ is absolutely continuous on $[a, b]$ and $\zeta \in[a, b]$. If $g: \Omega \rightarrow[a, b]$ is Lebesgue $\mu$-measurable on $\Omega$ such that $f \circ g, g,(g-\zeta)^{2} \in L(\Omega, \mu)$, with $\int_{\Omega} d \mu=1$, then

$$
\begin{gathered}
\int_{\Omega} f \circ g d \mu-f(\zeta)-\left(\int_{\Omega} g d \mu-\zeta\right) f^{\prime}(\zeta)-\frac{1}{2} \lambda \int_{\Omega}(g-\zeta)^{2} d \mu \\
=\int_{\Omega}(g-\zeta)^{2}\left(\int_{0}^{1}(1-s)\left[f^{\prime \prime}((1-s) \zeta+s g)-\lambda\right] d s\right) d \mu \\
=\int_{0}^{1}(1-s)\left(\int_{\Omega}(g-\zeta)^{2}\left[f^{\prime \prime}((1-s) \zeta+s g)-\lambda\right] d \mu\right) d s
\end{gathered}
$$

for any $\lambda \in \mathbb{C}$.

Proof Since $f^{\prime}$ is absolutely continuous function, $f^{\prime \prime}$ exists almost everywhere and by Taylor's formula with integral remainder we have

$$
f(x)=f(\zeta)+(x-\zeta) f^{\prime}(\zeta)+(x-\zeta)^{2} \int_{0}^{1}(1-s) f^{\prime \prime}((1-s) \zeta+s x) d s
$$

for any $\zeta, x \in[a, b]$. We observe that for $\lambda \in \mathbb{C}$ we have

$$
\begin{aligned}
& (x-\zeta)^{2} \int_{0}^{1}(1-s)\left[f^{\prime \prime}((1-s) \zeta+s x)-\lambda\right] d s \\
& =(x-\zeta)^{2} \int_{0}^{1}(1-s) f^{\prime \prime}((1-s) \zeta+s x) d s-(x-\zeta)^{2} \lambda \int_{0}^{1}(1-s) d s \\
& =(x-\zeta)^{2} \int_{0}^{1}(1-s) f^{\prime \prime}((1-s) \zeta+s x) d s-\frac{1}{2}(x-\zeta)^{2} \lambda
\end{aligned}
$$

and by (2.2) we get

$$
\begin{aligned}
f(x)= & f(\zeta)+(x-\zeta) f^{\prime}(\zeta)+\frac{1}{2} \lambda(x-\zeta)^{2} \\
& +(x-\zeta)^{2} \int_{0}^{1}(1-s)\left[f^{\prime \prime}((1-s) \zeta+s x)-\lambda\right] d s
\end{aligned}
$$

for any $\zeta, x \in[a, b]$ and $\lambda \in \mathbb{C}$. Now, if we replace $x$ with $g(t) \in[a, b]$ we get

$$
\begin{aligned}
f(g(t))= & f(\zeta)+(g(t)-\zeta) f^{\prime}(\zeta)+\frac{1}{2} \lambda(g(t)-\zeta)^{2} \\
& +(g(t)-\zeta)^{2} \int_{0}^{1}(1-s)\left[f^{\prime \prime}((1-s) \zeta+s g(t))-\lambda\right] d s
\end{aligned}
$$

for any $\zeta \in[a, b], t \in \Omega$ and $\lambda \in \mathbb{C}$. By integrating (2.4) on $\Omega$ and using the fact that $\int_{\Omega} d \mu=$ 1 , we obtain the first result in (2.1) by rearranging the terms. The second part follows by Fubini's theorem. 
We denote by $\sigma^{2}(g)$, the dispersion of a $\mu$-measurable function $g$ on $\Omega$, that is,

$$
\sigma^{2}(g):=\int_{\Omega} g^{2} d \mu-\left(\int_{\Omega} g d \mu\right)^{2}=\int_{\Omega}\left(g-\int_{\Omega} g d \mu\right)^{2} d \mu .
$$

In what follows, we have a particular case of Lemma 1.

Corollary 1 Under the assumptions of Lemma 1, we have the following identities when $\zeta=\int_{\Omega} g d \mu$ :

$$
\begin{aligned}
& \int_{\Omega} f \circ g d \mu-f\left(\int_{\Omega} g d \mu\right)-\frac{1}{2} \lambda \sigma^{2}(g) \\
& \quad=\int_{\Omega}\left(g-\int_{\Omega} g d \mu\right)^{2}\left(\int_{0}^{1}(1-s)\left[f^{\prime \prime}\left((1-s) \int_{\Omega} g d \mu+s g\right)-\lambda\right] d s\right) d \mu \\
& =\int_{0}^{1}(1-s)\left(\int_{\Omega}\left(g-\int_{\Omega} g d \mu\right)^{2}\left[f^{\prime \prime}\left((1-s) \int_{\Omega} g d \mu+s g\right)-\lambda\right] d \mu\right) d s
\end{aligned}
$$

for any $\lambda \in \mathbb{C}$.

Remark 1 Following the main idea of Costarelli and Spigler [2], one may obtain another estimates by considering the mean-value form of the remainder in (2.2)

$$
f(x)=f(\zeta)+(x-\zeta) f^{\prime}(\zeta)+\frac{1}{2} f^{\prime \prime}(\xi)(x-\zeta)^{2},
$$

where $\xi$ is between $x$ and $\zeta$. By setting $x=g(t)$, and integrate (2.6) on $\Omega$, we obtain

$$
\int_{\Omega} f \circ g d \mu=f(\zeta)+f^{\prime}(\zeta)\left(\int_{\Omega} g d \mu-\zeta\right)+\frac{1}{2} \int_{\Omega} f^{\prime \prime}(\xi)(g-\zeta)^{2} d \mu
$$

where $\xi=\xi(t)$ is between $g(t)$ and $\zeta$.

Let $\varphi: I \rightarrow \mathbb{R}$ be a real-valued convex function, where $I$ is a connected bounded set in $\mathbb{R}$, and $f:[0,1] \rightarrow I$ a real-valued nonnegative function where $f \in L^{1}(0,1)$. Suppose that $\varphi$ is a $C^{2}$ function. Set $f \equiv \varphi, g \equiv f$, and $\zeta=c=f\left(x_{0}\right)\left(x_{0}\right.$ can be chosen arbitrarily such that $\left.f\left(x_{0}\right) \in \stackrel{I}{I}\right)$ in $(2.7)$, we have

$$
\begin{aligned}
& \int_{0}^{1} \varphi(f(x)) d x \\
& \quad=\varphi(c)+\varphi^{\prime}(c) \int_{0}^{1}(f(x)-c) d x+\frac{1}{2} \int_{0}^{1} \varphi^{\prime \prime}\left(c^{*}(x)\right)(g(x)-c)^{2} d x
\end{aligned}
$$

where $c^{*}(x)$ is between $f(x)$ and $\zeta=f\left(x_{0}\right)$. This estimate is given in the paper by Costarelli and Spigler [2], p.2 to investigate the sharpness of the Jensen inequality ( $c f$. Proposition 1).

\section{Main results}

In this section, we present our main results on the Jensen-Ostrowski type inequalities for various cases. We start by introducing the following notation:

$$
\|k\|_{\Omega, p}:= \begin{cases}\left(\int_{\Omega}|k(t)|^{p} d \mu(t)\right)^{1 / p}, & p \geq 1, k \in L_{p}(\Omega, \mu), \\ {\operatorname{ess} \sup _{t \in \Omega}|k(t)|,} \quad p=\infty, k \in L_{\infty}(\Omega, \mu)\end{cases}
$$


and

$$
\|f\|_{[0,1], p}:= \begin{cases}\left(\int_{0}^{1}|f(s)|^{p} d s\right)^{1 / p}, & p \geq 1, f \in L_{p}([0,1]), \\ {\operatorname{ess} \sup _{s \in[0,1]}|f(s)|,} p=\infty, f \in L_{\infty}([0,1]) .\end{cases}
$$

We denote by $\ell$, the identity function on $[0,1]$, namely, $\ell(t)=t(t \in[0,1])$; and for $t \in \Omega$, $\zeta \in[a, b]$ and $\lambda \in \mathbb{C}$, we have

$$
\underset{s \in[0,1]}{\operatorname{ess} \sup }\left|f^{\prime \prime}((1-s) \zeta+s g(t))-\lambda\right|=\left\|f^{\prime \prime}((1-\ell) \zeta+\ell g)-\lambda\right\|_{[0,1], \infty} .
$$

We state the first of our main results, for which the bounds are given in terms of the p-norms.

Theorem 1 Let $f: I \rightarrow \mathbb{C}$ be a differentiable function on $\stackrel{I}{I} f^{\prime}:[a, b] \subset \stackrel{I}{\rightarrow} \mathbb{C}$ is absolutely continuous on $[a, b]$ and $\zeta \in[a, b]$. If $g: \Omega \rightarrow[a, b]$ is Lebesgue $\mu$-measurable on $\Omega$ such that $f \circ g, g,(g-\zeta)^{2} \in L(\Omega, \mu)$, with $\int_{\Omega} d \mu=1$, then for any $\lambda \in \mathbb{C}$,

$$
\begin{aligned}
& \left|\int_{\Omega} f \circ g d \mu-f(\zeta)-\left(\int_{\Omega} g d \mu-\zeta\right) f^{\prime}(\zeta)-\frac{1}{2} \lambda \int_{\Omega}(g-\zeta)^{2} d \mu\right| \\
& \quad \leq \frac{1}{2} \int_{\Omega}(g-\zeta)^{2}\left\|f^{\prime \prime}((1-\ell) \zeta+\ell g)-\lambda\right\|_{[0,1], \infty} d \mu \\
& \quad \leq\left\{\begin{array}{l}
\frac{1}{2}\left\|(g-\zeta)^{2}\right\|_{\Omega, \infty}\|\| f^{\prime \prime}((1-\ell) \zeta+\ell g)-\lambda\left\|_{[0,1], \infty}\right\|_{\Omega, 1}, \\
\frac{1}{2}\left\|(g-\zeta)^{2}\right\|_{\Omega, p}\|\| f^{\prime \prime}((1-\ell) \zeta+\ell g)-\lambda\left\|_{[0,1], \infty}\right\|_{\Omega, q}, \quad p>1, \frac{1}{p}+\frac{1}{q}=1 . \\
\frac{1}{2}\left\|(g-\zeta)^{2}\right\|_{\Omega, 1}\|\| f^{\prime \prime}((1-\ell) \zeta+\ell g)-\lambda\left\|_{[0,1], \infty}\right\|_{\Omega, \infty},
\end{array}\right.
\end{aligned}
$$

Proof Taking the modulus in (2.1), we have

$$
\begin{aligned}
& \left|\int_{\Omega} f \circ g d \mu-f(\zeta)-\left(\int_{\Omega} g d \mu-\zeta\right) f^{\prime}(\zeta)-\frac{1}{2} \lambda \int_{\Omega}(g-\zeta)^{2} d \mu\right| \\
& \quad \leq \int_{0}^{1}(1-s)\left(\int_{\Omega}(g-\zeta)^{2}\left|f^{\prime \prime}((1-s) \zeta+s g)-\lambda\right| d \mu\right) d s \\
& \quad \leq \int_{0}^{1}(1-s)\left(\int_{\Omega}(g-\zeta)^{2}\left\|f^{\prime \prime}((1-\ell) \zeta+\ell g)-\lambda\right\|_{[0,1], \infty} d \mu\right) d s \\
& \quad=\int_{0}^{1}(1-s) d s\left(\int_{\Omega}(g-\zeta)^{2}\left\|f^{\prime \prime}((1-\ell) \zeta+\ell g)-\lambda\right\|_{[0,1], \infty} d \mu\right) \\
& \quad=\frac{1}{2} \int_{\Omega}(g-\zeta)^{2}\left\|f^{\prime \prime}((1-\ell) \zeta+\ell g)-\lambda\right\|_{[0,1], \infty} d \mu .
\end{aligned}
$$

The proof is completed by utilising Hölder's inequality.

For the next result, we need the following notation and proposition: for $\gamma, \Gamma \in \mathbb{C}$ and $[a, b]$ an interval of real numbers, define the sets of complex-valued functions [9]

$$
\bar{U}_{[a, b]}(\gamma, \Gamma):=\{h:[a, b] \rightarrow \mathbb{C} \mid \operatorname{Re}[(\Gamma-h(t))(\overline{h(t)}-\bar{\gamma})] \geq 0 \text { for a.e. } t \in[a, b]\}
$$


and

$$
\bar{\Delta}_{[a, b]}(\gamma, \Gamma):=\left\{h:[a, b] \rightarrow \mathbb{C}|| h(t)-\frac{\gamma+\Gamma}{2}\left|\leq \frac{1}{2}\right| \Gamma-\gamma \mid \text { for a.e. } t \in[a, b]\right\} .
$$

The following representation results may be stated [9].

Proposition 5 For any $\gamma, \Gamma \in \mathbb{C}$ and $\gamma \neq \Gamma$, we have

(i) $\bar{U}_{[a, b]}(\gamma, \Gamma)$ and $\bar{\Delta}_{[a, b]}(\gamma, \Gamma)$ are nonempty, convex and closed sets;

(ii) $\bar{U}_{[a, b]}(\gamma, \Gamma)=\bar{\Delta}_{[a, b]}(\gamma, \Gamma)$;

(iii) $\bar{U}_{[a, b]}(\gamma, \Gamma)=\{h:[a, b] \rightarrow \mathbb{C} \mid(\operatorname{Re}(\Gamma)-\operatorname{Re}(h(t)))(\operatorname{Re}(h(t))-\operatorname{Re}(\gamma))+(\operatorname{Im}(\Gamma)-$ $\operatorname{Im}(h(t)))(\operatorname{Im}(h(t))-\operatorname{Im}(\gamma)) \geq 0$ for a.e. $t \in[a, b]\}$.

We have the following Jensen-Ostrowski inequality for functions with bounded second derivatives.

Theorem 2 Let $f: I \rightarrow \mathbb{C}$ be a differentiable function on $\stackrel{\circ}{I}, f^{\prime}:[a, b] \subset \stackrel{\circ}{I} \rightarrow \mathbb{C}$ is absolutely continuous on $[a, b]$ and $\zeta \in[a, b]$. For some $\gamma, \Gamma \in \mathbb{C}, \gamma \neq \Gamma$, assume that $f^{\prime \prime} \in \bar{U}_{[a, b]}(\gamma, \Gamma)=$ $\bar{\Delta}_{[a, b]}(\gamma, \Gamma)$. If $g: \Omega \rightarrow[a, b]$ is Lebesgue $\mu$-measurable on $\Omega$ such that $f \circ g, g,(g-\zeta)^{2} \in$ $L(\Omega, \mu)$, with $\int_{\Omega} d \mu=1$, then

$$
\begin{aligned}
& \left|\int_{\Omega}(f \circ g) d \mu-f(\zeta)-\left(\int_{\Omega} g d \mu-\zeta\right) f^{\prime}(\zeta)-\frac{\gamma+\Gamma}{4} \int_{\Omega}(g-\zeta)^{2} d \mu\right| \\
& \quad \leq \frac{1}{4}|\Gamma-\gamma|\left[\sigma^{2}(g)+\left(\int_{\Omega} g d \mu-\zeta\right)^{2}\right] .
\end{aligned}
$$

In particular, we have the following Ostrowski type inequality:

$$
\begin{aligned}
& \mid \int_{\Omega}(f \circ g) d \mu-f\left(\frac{a+b}{2}\right) \\
& \quad-\left(\int_{\Omega} g d \mu-\frac{a+b}{2}\right) f^{\prime}\left(\frac{a+b}{2}\right)-\frac{\gamma+\Gamma}{4} \int_{\Omega}\left(g-\frac{a+b}{2}\right)^{2} d \mu \mid \\
& \quad \leq \frac{1}{4}|\Gamma-\gamma|\left[\sigma^{2}(g)+\left(\int_{\Omega} g d \mu-\frac{a+b}{2}\right)^{2}\right],
\end{aligned}
$$

and we have the following Jensen type inequality:

$$
\left|\int_{\Omega}(f \circ g) d \mu-f\left(\int_{\Omega} g d \mu\right)-\frac{\gamma+\Gamma}{4} \sigma^{2}(g)\right| \leq \frac{1}{4}|\Gamma-\gamma| \sigma^{2}(g) .
$$

Proof By equality (2.1), for $\lambda=\frac{\gamma+\Gamma}{2}$ we have

$$
\begin{gathered}
\int_{\Omega}(f \circ g) d \mu-f(\zeta)-\left(\int_{\Omega} g d \mu-\zeta\right) f^{\prime}(\zeta)-\frac{\gamma+\Gamma}{4} \int_{\Omega}(g-\zeta)^{2} d \mu \\
=\int_{\Omega}\left[(g-\zeta)^{2} \int_{0}^{1}(1-s)\left[f^{\prime \prime}((1-s) \zeta+s g)-\frac{\gamma+\Gamma}{2}\right] d s\right] d \mu .
\end{gathered}
$$

Since $f^{\prime \prime} \in \bar{\Delta}_{[a, b]}(\gamma, \Gamma)$, we have

$$
\left|f^{\prime \prime}((1-s) \zeta+s g)-\frac{\gamma+\Gamma}{2}\right| \leq \frac{1}{2}|\Gamma-\gamma|
$$


for almost every $s \in[0,1]$ and any $t \in \Omega$. Multiply (3.7) with $s>0$ and integrate over [0,1], we obtain

$$
\int_{0}^{1}(1-s)\left|f^{\prime \prime}((1-s) \zeta+s g)-\frac{\gamma+\Gamma}{2}\right| d s \leq \frac{1}{2}|\Gamma-\gamma| \int_{0}^{1}(1-s) d s=\frac{1}{4}|\Gamma-\gamma|
$$

for any $t \in \Omega$.

Taking the modulus of (3.6), we get the following, by (3.8):

$$
\begin{aligned}
& \left|\int_{\Omega}(f \circ g) d \mu-f(\zeta)-\left(\int_{\Omega} g d \mu-\zeta\right) f^{\prime}(\zeta)-\frac{\gamma+\Gamma}{4} \int_{\Omega}(g-\zeta)^{2} d \mu\right| \\
& \quad \leq \int_{\Omega}\left[(g-\zeta)^{2} \int_{0}^{1}(1-s)\left|f^{\prime \prime}((1-s) \zeta+s g)-\frac{\gamma+\Gamma}{2}\right| d s\right] d \mu \\
& \quad \leq \frac{1}{4}|\Gamma-\gamma| \int_{\Omega}(g-\zeta)^{2} d \mu,
\end{aligned}
$$

and the proof is completed. We also note that

$$
\begin{aligned}
\int_{\Omega}(g-\zeta)^{2} d \mu & =\int_{\Omega}\left(g-\int_{\Omega} g d \mu+\int_{\Omega} g d \mu-\zeta\right)^{2} d \mu \\
& =\int_{\Omega}\left(g-\int_{\Omega} g d \mu\right)^{2} d \mu+\left(\int_{\Omega} g d \mu-\zeta\right)^{2} \\
& =\sigma^{2}(g)+\left(\int_{\Omega} g d \mu-\zeta\right)^{2}
\end{aligned}
$$

We obtain (3.4) and (3.5), by setting $\zeta=(a+b) / 2$ and $\zeta=\int_{\Omega} g d \mu$, respectively.

Remark 2 If $f^{\prime}$ is convex in Theorem 2, then $\gamma=f_{+}^{\prime \prime}(a)$ and $\Gamma=f_{-}^{\prime \prime}(b)$.

We recall the following definition.

Definition 1 Let $h: I \subset \mathbb{R} \rightarrow \mathbb{R}$ be a real-valued function. Then:

(1) $h$ is convex, if for any $x, y \in I$ and $s \in[0,1]$, we have

$$
h((1-s) x+s y) \leq(1-s) h(x)+\operatorname{sh}(y) .
$$

(2) $h$ is quasi-convex, if for any $x, y \in I$ and $s \in[0,1]$, we have

$$
h((1-s) x+s y) \leq \max \{h(x), h(y)\} .
$$

(3) $h$ is log-convex, if for any $x, y \in I$ and $s \in[0,1]$, we have

$$
h((1-s) x+s y) \leq h(x)^{1-s} h(y)^{s} .
$$

(4) For a fixed $q \in(0,1], h$ is $q$-convex, if for any $x, y \in I$ and $s \in[0,1]$, we have

$$
h((1-s) x+s y) \leq(1-s)^{q} h(x)+s^{q} h(y) .
$$


We refer the reader to the paper by Dragomir [13], for further background on these notions of convexity.

In the next theorem, we assume that $\left|f^{\prime \prime}\right|$ satisfies some convexity properties.

Theorem 3 Let $f: I \rightarrow \mathbb{C}$ be a differentiable function on $I, f^{\prime}:[a, b] \subset I \rightarrow \mathbb{C}$ is absolutely continuous on $[a, b]$ and $\zeta \in[a, b]$. Suppose that $g: \Omega \rightarrow[a, b]$ is Lebesgue $\mu$-measurable on $\Omega$ such that $f \circ g, g,(g-\zeta)^{2} \in L(\Omega, \mu)$, with $\int_{\Omega} d \mu=1$.

(i) If $\left|f^{\prime \prime}\right|$ is convex, then we have

$$
\begin{aligned}
& \left|\int_{\Omega}(f \circ g) d \mu-f(\zeta)-\left(\int_{\Omega} g d \mu-\zeta\right) f^{\prime}(\zeta)\right| \\
& \quad \leq \frac{1}{3}\left[\left|f^{\prime \prime}(\zeta)\right| \int_{\Omega}(g-\zeta)^{2} d \mu+\frac{1}{2} \int_{\Omega}(g-\zeta)^{2}\left|f^{\prime \prime} \circ g\right| d \mu\right] .
\end{aligned}
$$

(ii) If $\left|f^{\prime \prime}\right|$ is quasi-convex, then we have

$$
\begin{aligned}
& \left|\int_{\Omega}(f \circ g) d \mu-f(\zeta)-\left(\int_{\Omega} g d \mu-\zeta\right) f^{\prime}(\zeta)\right| \\
& \quad \leq \frac{1}{2} \int_{\Omega}(g-\zeta)^{2} \max \left\{\left|f^{\prime \prime}(\zeta)\right|,\left|f^{\prime \prime} \circ g\right|\right\} d \mu .
\end{aligned}
$$

(iii) If $\left|f^{\prime \prime}\right|$ is log-convex, then we have

$$
\begin{aligned}
& \left|\int_{\Omega}(f \circ g) d \mu-f(\zeta)-\left(\int_{\Omega} g d \mu-\zeta\right) f^{\prime}(\zeta)\right| \\
& \quad \leq \int_{\Omega}(g-\zeta)^{2} \\
& \quad \times\left|\frac{-\left|f^{\prime \prime}(\zeta)\right|+\left|f^{\prime \prime} \circ g\right|+\left|f^{\prime \prime}(\zeta)\right|\left[\log \left(\left|f^{\prime \prime}(\zeta)\right|\right)-\log \left(\left|f^{\prime \prime} \circ g\right|\right)\right]}{\left[\log \left(\left|f^{\prime \prime}(\zeta)\right|\right)-\log \left(\left|f^{\prime \prime} \circ g\right|\right)\right]^{2}}\right| d \mu .
\end{aligned}
$$

(iv) If $\left|f^{\prime \prime}\right|$ is $q$-convex (for a fixed $\left.q \in(0,1]\right)$, then we have

$$
\begin{aligned}
& \left|\int_{\Omega}(f \circ g) d \mu-f(\zeta)-\left(\int_{\Omega} g d \mu-\zeta\right) f^{\prime}(\zeta)\right| \\
& \quad \leq \frac{1}{(q+2)}\left[\left|f^{\prime \prime}(\zeta)\right| \int_{\Omega}(g-\zeta)^{2} d \mu+\frac{1}{q+1} \int_{\Omega}(g-\zeta)^{2}\left|f^{\prime \prime} \circ g\right| d \mu\right]
\end{aligned}
$$

Proof (i) If $\left|f^{\prime \prime}\right|$ is convex, then

$$
\left|f^{\prime \prime}((1-s) \zeta+s g(t))\right| \leq(1-s)\left|f^{\prime \prime}(\zeta)\right|+s\left|f^{\prime \prime}(g(t))\right| \quad \text { for all } t \in \Omega
$$

which implies that

$$
\begin{aligned}
& \int_{0}^{1}(1-s)\left|f^{\prime \prime}((1-s) \zeta+s g(t))\right| d s \\
& \quad \leq\left[\int_{0}^{1}(1-s)^{2} d s\right]\left|f^{\prime \prime}(\zeta)\right|+\left[\int_{0}^{1} s(1-s) d s\right]\left|f^{\prime \prime}(g(t))\right| \\
& \quad=\frac{1}{3}\left|f^{\prime \prime}(\zeta)\right|+\frac{1}{6}\left|f^{\prime \prime}(g(t))\right| \quad \text { for all } t \in \Omega .
\end{aligned}
$$


Crone et al. Journal of Inequalities and Applications ( 2015) 2015:328

Page 10 of 20

Thus,

$$
\begin{aligned}
& \int_{\Omega}\left|(g-\zeta)^{2} \int_{0}^{1}(1-s)\left[f^{\prime \prime}((1-s) \zeta+s g(t))\right] d s\right| d \mu \\
& \quad \leq \frac{1}{3}\left|f^{\prime \prime}(\zeta)\right| \int_{\Omega}(g-\zeta)^{2} d \mu+\frac{1}{6} \int_{\Omega}(g-\zeta)^{2}\left|f^{\prime \prime} \circ g\right| d \mu .
\end{aligned}
$$

The proof is completed by (2.1) with $\lambda=0$.

(ii) If $\left|f^{\prime \prime}\right|$ is quasi-convex, then

$$
\left|f^{\prime \prime}((1-s) \zeta+s g(t))\right| \leq \max \left\{\left|f^{\prime \prime}(\zeta)\right|,\left|f^{\prime \prime}(g(t))\right|\right\} \quad \text { for all } t \in \Omega
$$

which implies that

$$
\begin{aligned}
& \int_{0}^{1}(1-s)\left|f^{\prime \prime}((1-s) \zeta+s g(t))\right| d s \\
& \quad \leq\left[\int_{0}^{1}(1-s) d s\right] \max \left\{\left|f^{\prime \prime}(\zeta)\right|,\left|f^{\prime \prime}(g(t))\right|\right\} \\
& \quad=\frac{1}{2} \max \left\{\left|f^{\prime \prime}(\zeta)\right|,\left|f^{\prime \prime}(g(t))\right|\right\} \quad \text { for all } t \in \Omega .
\end{aligned}
$$

Thus,

$$
\begin{gathered}
\int_{\Omega}\left|(g-\zeta)^{2} \int_{0}^{1}(1-s)\left[f^{\prime \prime}((1-s) \zeta+s g(t))\right] d s\right| d \mu \\
\quad \leq \frac{1}{2} \int_{\Omega}(g-\zeta)^{2} \max \left\{\left|f^{\prime \prime}(\zeta)\right|,\left|f^{\prime \prime} \circ g\right|\right\} d \mu .
\end{gathered}
$$

The proof is completed by (2.1) with $\lambda=0$.

(iii) If $\left|f^{\prime \prime}\right|$ is log-convex, then

$$
\left|f^{\prime \prime}((1-s) \zeta+s g(t))\right| \leq\left|f^{\prime \prime}(\zeta)\right|^{1-s}\left|f^{\prime \prime}(g(t))\right|^{s} \quad \text { for all } t \in \Omega
$$

which implies that

$$
\begin{aligned}
& \int_{0}^{1}(1-s)\left|f^{\prime \prime}((1-s) \zeta+s g(t))\right| d s \\
& \quad \leq\left[\int_{0}^{1}(1-s)\left|f^{\prime \prime}(\zeta)\right|^{1-s}\left|f^{\prime \prime}(g(t))\right|^{s} d s\right] \\
& \quad=\frac{-\left|f^{\prime \prime}(\zeta)\right|+\left|f^{\prime \prime}(g(t))\right|+\left|f^{\prime \prime}(\zeta)\right|\left[\log \left(\left|f^{\prime \prime}(\zeta)\right|\right)-\log \left(\left|f^{\prime \prime}(g(t))\right|\right)\right]}{\left[\log \left(\left|f^{\prime \prime}(\zeta)\right|\right)-\log \left(\left|f^{\prime \prime}(g(t))\right|\right)\right]^{2}}
\end{aligned}
$$

for all $t \in \Omega$. Thus,

$$
\begin{aligned}
& \int_{\Omega}\left|(g-\zeta)^{2} \int_{0}^{1}(1-s)\left[f^{\prime \prime}((1-s) \zeta+s g(t))\right] d s\right| d \mu \\
& \quad \leq \int_{\Omega}(g-\zeta)^{2}\left|\frac{-\left|f^{\prime \prime}(\zeta)\right|+\left|f^{\prime \prime} \circ g\right|+\left|f^{\prime \prime}(\zeta)\right|\left[\log \left(\left|f^{\prime \prime}(\zeta)\right|\right)-\log \left(\left|f^{\prime \prime} \circ g\right|\right)\right]}{\left[\log \left(\left|f^{\prime \prime}(\zeta)\right|\right)-\log \left(\left|f^{\prime \prime} \circ g\right|\right)\right]^{2}}\right| d \mu .
\end{aligned}
$$

The proof is completed by (2.1) with $\lambda=0$. 
(iv) If $\left|f^{\prime \prime}\right|$ is $q$-convex (for a fixed $\left.q \in(0,1]\right)$, then

$$
\left|f^{\prime \prime}((1-s) \zeta+s g)\right| \leq(1-s)^{q}\left|f^{\prime \prime}(\zeta)\right|+s^{q}\left|f^{\prime \prime}(g(t))\right| \quad \text { for all } t \in \Omega
$$

which implies that

$$
\begin{aligned}
& \int_{0}^{1}(1-s)\left|f^{\prime \prime}((1-s) \zeta+s g(t))\right| d s \\
& \quad \leq\left[\int_{0}^{1}(1-s)^{q+1} d s\right]\left|f^{\prime \prime}(\zeta)\right|+\left[\int_{0}^{1}(1-s) s^{q} d s\right]\left|f^{\prime \prime}(g(t))\right| \\
& \quad=\frac{1}{q+2}\left|f^{\prime \prime}(\zeta)\right|+\frac{1}{(q+1)(q+2)}\left|f^{\prime \prime}(g(t))\right| \quad \text { for all } t \in \Omega .
\end{aligned}
$$

Thus,

$$
\begin{aligned}
& \int_{\Omega}\left|(g-\zeta)^{2} \int_{0}^{1}(1-s)\left[f^{\prime \prime}((1-s) \zeta+s g(t))\right] d s\right| d \mu \\
& \quad \leq \frac{1}{q+2}\left|f^{\prime \prime}(\zeta)\right| \int_{\Omega}(g-\zeta)^{2} d \mu+\frac{1}{(q+1)(q+2)} \int_{\Omega}(g-\zeta)^{2}\left|f^{\prime \prime} \circ g\right| d \mu .
\end{aligned}
$$

The proof is completed by (2.1) with $\lambda=0$.

\section{Generalised Ostrowski's inequality and bounds for the discrepancy in Jensen's inequality}

In this section, we provide a generalised version of the Ostrowski inequality (1.8) of Proposition 3, as well as bounds for the discrepancy in Jensen's integral inequality. We start with the following theorem.

Theorem 4 Let $f: I \rightarrow \mathbb{C}$ be a differentiable function on $\stackrel{\circ}{I}, f^{\prime}:[a, b] \subset \stackrel{\circ}{I} \rightarrow \mathbb{C}$ is absolutely continuous on $[a, b]$ and $\zeta \in[a, b]$. If $g: \Omega \rightarrow[a, b]$ is Lebesgue $\mu$-measurable on $\Omega$ such that $f \circ g, g,(g-\zeta)^{2} \in L(\Omega, \mu)$, with $\int_{\Omega} d \mu=1$, then we have the following Ostrowski type inequality:

$$
\begin{aligned}
& \left|\int_{\Omega}(f \circ g) d \mu-f(\zeta)-\left(\int_{\Omega} g d \mu-\zeta\right) f^{\prime}(\zeta)\right| \\
& \quad \leq \frac{1}{2}\left\|f^{\prime \prime}\right\|_{[a, b], \infty}\left[\sigma^{2}(g)+\left(\int_{\Omega} g d \mu-\zeta\right)^{2}\right] .
\end{aligned}
$$

We also have the following Jensen type inequality:

$$
\left|\int_{\Omega}(f \circ g) d \mu-f\left(\int_{\Omega} g d \mu\right)\right| \leq \frac{1}{2}\left\|f^{\prime \prime}\right\|_{[a, b], \infty} \sigma^{2}(g),
$$

which is the best inequality one can get from (4.1).

Proof We have from (3.1) with $\lambda=0$,

$$
\begin{aligned}
& \left|\int_{\Omega}(f \circ g) d \mu-f(\zeta)-\left(\int_{\Omega} g d \mu-\zeta\right) f^{\prime}(\zeta)\right| \\
& \quad \leq \frac{1}{2} \int_{\Omega}(g-\zeta)^{2}\left\|f^{\prime \prime}((1-\ell) \zeta+\ell g)\right\|_{[0,1], \infty} d \mu .
\end{aligned}
$$


For any $t \in \Omega$ and almost every $s \in[0,1]$, we have

$$
\left|f^{\prime \prime}((1-s) \zeta+s g(t))\right| \leq \underset{u \in[a, b]}{\operatorname{ess} \sup }\left|f^{\prime \prime}(u)\right|=\left\|f^{\prime \prime}\right\|_{[a, b], \infty}
$$

which implies that

$$
\left\|f^{\prime \prime}((1-\ell) \zeta+\ell g)\right\|_{[0,1], \infty}=\underset{s \in[0,1]}{\operatorname{ess} \sup }\left|f^{\prime \prime}((1-s) \zeta+s g(t))\right| \leq\left\|f^{\prime \prime}\right\|_{[a, b], \infty}
$$

Therefore, we have

$$
\left|\int_{\Omega}(f \circ g) d \mu-f(\zeta)-\left(\int_{\Omega} g d \mu-\zeta\right) f^{\prime}(\zeta)\right| \leq \frac{1}{2}\left\|f^{\prime \prime}\right\|_{[a, b], \infty} \int_{\Omega}(g-\zeta)^{2} d \mu .
$$

This proves (4.1). Note the use of (3.9). By choosing $\zeta=\int_{\Omega} g d \mu$ in (4.1), we obtain (4.2).

Remark 3 (Ostrowski type inequality) We recall the quantity

$$
\int_{\Omega}(g-\zeta)^{2} d \mu=\int_{\Omega}\left(g-\int_{\Omega} g d \mu\right)^{2} d \mu+\left(\int_{\Omega} g d \mu-\zeta\right)^{2}
$$

In the case that $\Omega=[a, b], g:[a, b] \rightarrow[a, b]$ is defined by $g(t)=t$ and $\mu(t)=\frac{t}{b-a}$, we have

$$
\int_{\Omega} g d \mu=\frac{1}{b-a} \int_{a}^{b} t d t=\frac{a+b}{2}
$$

and (4.3) becomes

$$
\begin{aligned}
& \int_{\Omega}\left(g-\int_{\Omega} g d \mu\right)^{2} d \mu+\left(\int_{\Omega} g d \mu-\zeta\right)^{2} \\
& =\frac{1}{b-a} \int_{a}^{b}\left(t-\frac{a+b}{2}\right)^{2} d t+\left(\zeta-\frac{a+b}{2}\right)^{2} \\
& =\frac{(b-a)^{2}}{12}+\left(\zeta-\frac{a+b}{2}\right)^{2}
\end{aligned}
$$

Under this assumption, the left-hand side of (4.1) becomes

$$
\left|\frac{1}{b-a} \int_{a}^{b} f(t) d t-f(\zeta)-\left(\frac{a+b}{2}-\zeta\right) f^{\prime}(\zeta)\right|
$$

and the right-hand side of (4.1) becomes

$$
\begin{gathered}
\frac{1}{2}\left\|f^{\prime \prime}\right\|_{[a, b], \infty}\left[\int_{\Omega}\left(g-\int_{\Omega} g d \mu\right)^{2} d \mu+\left(\int_{\Omega} g d \mu-\zeta\right)^{2}\right] \\
=\frac{1}{2}\left\|f^{\prime \prime}\right\|_{[a, b], \infty}\left[\frac{1}{12}(b-a)^{2}+\left(\zeta-\frac{a+b}{2}\right)^{2}\right] .
\end{gathered}
$$

Thus, (4.1) becomes

$$
\left|\frac{1}{b-a} \int_{a}^{b} f(t) d t-f(\zeta)+\left(\zeta-\frac{a+b}{2}\right) f^{\prime}(\zeta)\right| \leq\left[\frac{1}{24}+\frac{1}{2} \frac{\left(\zeta-\frac{a+b}{2}\right)^{2}}{(b-a)^{2}}\right](b-a)^{2}\left\|f^{\prime \prime}\right\|_{[a, b], \infty}
$$


for $\zeta \in[a, b]$, which recovers the result by Cerone et al. [6] (cf. Dragomir and Rassias [8], p.87), by multiplying the above inequality by $(b-a)$ and setting $\zeta=x \in[a, b]$. We conclude that the Ostrowski inequality (4.1) is a generalised version of (1.8) in a measure-theoretic (probabilistic) form.

Remark 4 We may obtained the results in Theorem 4 from (2.7), which uses the meanvalue form of the remainder, so that

$$
\begin{aligned}
& \left|\int_{\Omega} f \circ g d \mu-f(\zeta)-f^{\prime}(\zeta)\left(\int_{\Omega} g d \mu-\zeta\right)\right| \\
& \quad \leq \frac{1}{2} \int_{\Omega}\left|f^{\prime \prime}(\xi)\right|(g-\zeta)^{2} d \mu \\
& \quad \leq \frac{1}{2}\left\|f^{\prime \prime}\right\|_{[a, b], \infty} \int_{\Omega}(g-\zeta)^{2} d \mu \\
& \quad=\frac{1}{2}\left\|f^{\prime \prime}\right\|_{[a, b], \infty}\left(\sigma^{2}(g)+\left(\int_{\Omega} g d \mu-\zeta\right)^{2}\right) .
\end{aligned}
$$

Let $\varphi: I \rightarrow \mathbb{R}$ be a real-valued convex function, where $I$ is a connected bounded set in $\mathbb{R}$ and $f:[0,1] \rightarrow I$ a real-valued nonnegative function where $f \in L^{1}(0,1)$. Suppose that $\varphi$ is a $C^{2}$ function. Set $f \equiv \varphi, g \equiv f$, and $\zeta=\int_{0}^{1} g(t) d t$ in (4.4), we have

$$
\begin{aligned}
& \left|\int_{0}^{1} \varphi(f(x)) d x-\varphi\left(\int_{0}^{1} f(x) d x\right)\right| \\
& \quad \leq \frac{1}{2}\left\|\varphi^{\prime \prime}\right\|_{I_{2}, \infty} \int_{0}^{1}\left(f(x)-\int_{0}^{1} f(x) d x\right)^{2} d t,
\end{aligned}
$$

where $I_{2}$ is the domain of $\varphi^{\prime \prime}$. Furthermore, if $\varphi$ is convex and $f$ is continuous, then the mean-value theorem for integration asserts that there exists $x_{0} \in[0,1]$ such that $\int_{0}^{1} f(t) d t=f\left(x_{0}\right)=: c$, and thus

$$
\begin{aligned}
0 & \leq \int_{0}^{1} \varphi(f(x)) d x-\varphi\left(\int_{0}^{1} f(x) d x\right) \\
& \leq \frac{1}{2}\left\|\varphi^{\prime \prime}\right\|_{I_{2}, \infty} \int_{0}^{1}(f(x)-c)^{2} d x \\
& =\frac{1}{2}\left\|\varphi^{\prime \prime}\right\|_{I_{2}, \infty}\|f-c\|_{[0,1], 2}^{2} \\
& \leq \frac{1}{2}\left\|\varphi^{\prime \prime}\right\|_{I_{2}, \infty}\left(\|f-c\|_{[0,1], 2}^{2}+\|f-c\|_{[0,1], 1}^{2}\right),
\end{aligned}
$$

where the last estimate is given by Costarelli and Spigler in (1.5). Here, our result is shown to be sharper than the result by Costarelli and Spigler (1.5). When $\varphi$ is assumed to be $C^{2}$-smooth, the result (1.6) by Costarelli and Spigler is sharper than our estimate:

$$
\begin{aligned}
0 & \leq \int_{0}^{1} \varphi(f(x)) d x-\varphi\left(\int_{0}^{1} f(x) d x\right) \\
& \leq \frac{1}{2}\left\|\varphi^{\prime \prime}\right\|_{I_{2}, \infty}\|f-c\|_{[0,1], 2}^{2}-\frac{1}{2} \inf _{I_{2}} \varphi^{\prime \prime}\left[\int_{0}^{1}(f(x)-c) d x\right]^{2} \\
& \leq \frac{1}{2}\left\|\varphi^{\prime \prime}\right\|_{I_{2}, \infty}\|f-c\|_{[0,1], 2}^{2} .
\end{aligned}
$$


Costarelli and Spigler provide an example to compare the two bounds given in (1.5) and (1.6) [2], Example 3.1, p.5. In what follows, we recall the example and provide a comparison to the bound obtained in (4.5). Let $\varphi(y)=-\sin \pi y$ and $f(x)=x^{2}$. The true discrepancy $E:=$ $\int_{0}^{1} \varphi(f(x)) d x-\varphi\left(\int_{0}^{1} f(x) d x\right)$ between the two sides of the Jensen inequality is $E \approx 0.3612$. Using (1.5), the estimate for $E$ is: $E \leq 1.3627 \ldots$ Noting that $\inf _{I_{2}} \varphi^{\prime \prime}\left[\int_{0}^{1}(f(x)-c) d x\right]^{2}=0$, the estimate for $E$ by using (1.6) is the same as that of (4.5), which is closer to the true discrepancy, that is,

$$
E \leq \frac{1}{2}\left\|\varphi^{\prime \prime}\right\|_{I_{2}, \infty}\|f-c\|_{[0,1], 2}^{2}=\frac{\pi^{2}}{2}\left[\frac{1}{9}-\frac{2}{9}+\frac{1}{5}\right] \approx 0.4386
$$

Remark 5 The assumption of convexity on $\left|f^{\prime \prime}\right|$ provides refinements for (4.2) (cf. Theorem 4), as shown in the following: If $\left|f^{\prime \prime}\right|$ is convex, then

$$
\begin{aligned}
& \left|\int_{\Omega}(f \circ g) d \mu-f\left(\int_{\Omega} g d \mu\right)\right| \\
& \quad \leq \frac{1}{3}\left[\left|f^{\prime \prime}\left(\int_{\Omega} g d \mu\right)\right| \sigma^{2}(g)+\frac{1}{2} \int_{\Omega}\left(g-\int_{\Omega} g d \mu\right)^{2}\left|f^{\prime \prime} \circ g\right| d \mu\right] \\
& \quad \leq \frac{1}{3}\left[\left\|f^{\prime \prime}\right\|_{[a, b], \infty} \sigma^{2}(g)+\frac{1}{2}\left\|f^{\prime \prime}\right\|_{[a, b], \infty} \int_{\Omega}\left(g-\int_{\Omega} g d \mu\right)^{2} d \mu\right] \\
& \quad=\frac{1}{2}\left\|f^{\prime \prime}\right\|_{[a, b], \infty} \sigma^{2}(g) .
\end{aligned}
$$

We give an example to the above comparison. Let $f(t)=e^{-t}$ and $g(t)=t$ for $t \in[1,2]$. The true discrepancy in the Jensen inequality is

$$
E=\left|\int_{1}^{2} e^{-t} d t-f\left(\int_{1}^{2} t d t\right)\right|=\frac{e-1}{e^{2}}-e^{-3 / 2} \approx 0.009414
$$

The estimate for the discrepancy given by Theorem 4 is

$$
E \leq \frac{1}{2} \max \left\{e^{-t}, t \in[1,2]\right\} \int_{1}^{2}\left(t-\frac{3}{2}\right)^{2} d t=\frac{1}{24} e^{-1} \approx 0.015328
$$

The estimate for the discrepancy given by Theorem 3 is closer to the true discrepancy, that is,

$$
\begin{aligned}
E & \leq \frac{1}{3}\left[\left|f^{\prime \prime}\left(\frac{3}{2}\right)\right| \int_{1}^{2}\left(t-\frac{3}{2}\right)^{2} d t+\frac{1}{2} \int_{1}^{2}\left(t-\frac{3}{2}\right)^{2} e^{-t} d t\right] \\
& =\frac{1}{3}\left[\frac{1}{12} e^{-3 / 2}+\frac{1}{2}\left(\frac{5 e-13}{4 e^{2}}\right)\right] \\
& \approx 0.009533 .
\end{aligned}
$$

\section{Applications for $f$-divergence}

In the same spirit to that of [12], we apply our result to obtain inequalities for $f$-divergence measures. Assume that a set $\Omega$ and the $\sigma$-finite measure $\mu$ are given. Consider the set of 
all probability densities on $\mu$ to be

$$
\mathcal{P}:=\left\{p \mid p: \Omega \rightarrow \mathbb{R}, p(t) \geq 0, \int_{\Omega} p(t) d \mu(t)=1\right\} .
$$

We recall the definition of some divergence measures which we use in this text. For other divergence measures, we refer the readers to [14-18] and [19]. The Kullback-Leibler divergence [20] is defined as

$$
D_{K L}(p, q):=\int_{\Omega} p(t) \log \left[\frac{p(t)}{q(t)}\right] d \mu(t), \quad p, q \in \mathcal{P} .
$$

The following is the definition of the $\chi^{2}$-divergence:

$$
D_{\chi^{2}}(p, q):=\int_{\Omega} p(t)\left[\left(\frac{q(t)}{p(t)}\right)^{2}-1\right] d \mu(t), \quad p, q \in \mathcal{P} .
$$

The Csiszár $f$-divergence is defined as follows [21]:

$$
I_{f}(p, q):=\int_{\Omega} p(t) f\left[\frac{q(t)}{p(t)}\right] d \mu(t), \quad p, q \in \mathcal{P}
$$

where $f$ is convex on $(0, \infty)$. It is assumed that $f(u)$ is zero and strictly convex at $u=1$. The Kullback-Leibler divergence and the $\chi^{2}$-divergence are particular instances of the Csiszár $f$-divergence. For the basic properties of the Csiszár $f$-divergence, we refer the reader to $[21,22]$ and [23].

Proposition 6 Let $f:(0, \infty) \rightarrow \mathbb{R}$ be a differentiable convex function with the property that $f(1)=0$. Assume that $p, q \in \mathcal{P}$ and there exists constants $0<r<1<R<\infty$ such that

$$
r \leq \frac{q(t)}{p(t)} \leq R \quad \text { for } \mu \text {-a.e. } t \in \Omega \text {. }
$$

If $\zeta \in[r, R]$ and $f^{\prime}$ is absolutely continuous on $[r, R]$, then we have the inequalities

$$
\left|I_{f}(p, q)-f(\zeta)-(1-\zeta) f^{\prime}(\zeta)\right| \leq \frac{1}{2}\left\|f^{\prime \prime}\right\|_{[r, R], \infty}\left[D_{\chi^{2}}(p, q)+(\zeta-1)^{2}\right] .
$$

In particular, by choosing $\zeta=(r+R) / 2$, we have

$$
\begin{gathered}
\left|I_{f}(p, q)-f\left(\frac{r+R}{2}\right)-\left(1-\frac{r+R}{2}\right) f^{\prime}\left(\frac{r+R}{2}\right)\right| \\
\quad \leq \frac{1}{2}\left\|f^{\prime \prime}\right\|_{[r, R], \infty}\left[D_{\chi^{2}}(p, q)+\left(\frac{r+R}{2}-1\right)^{2}\right],
\end{gathered}
$$

and when $\zeta=1$,

$$
\left|I_{f}(p, q)\right| \leq \frac{1}{2}\left\|f^{\prime \prime}\right\|_{[r, R], \infty} D_{\chi^{2}}(p, q) .
$$


Proof We choose $g(t)=q(t) / p(t)$ and noting that $\int_{\Omega} p(t) d \mu=1$, in inequality (4.1), we have

$$
\begin{aligned}
& \left|\int_{\Omega} f\left(\frac{q(t)}{p(t)}\right) p(t) d \mu-f(\zeta)-\left(\int_{\Omega} q(t) d \mu-\zeta\right) f^{\prime}(\zeta)\right| \\
& \quad=\left|I_{f}(p, q)-f(\zeta)-(1-\zeta) f^{\prime}(\zeta)\right| \\
& \quad \leq \frac{1}{2}\left\|f^{\prime \prime}\right\|_{[r, R], \infty}\left[\int_{\Omega}\left(\frac{q(t)}{p(t)}-\int_{\Omega} q(t) d \mu\right)^{2} p(t) d \mu+\left(\int_{\Omega} q(t) d \mu-\zeta\right)^{2}\right] \\
& \quad=\frac{1}{2}\left\|f^{\prime \prime}\right\|_{[r, R], \infty}\left[\int_{\Omega}\left(\frac{q(t)}{p(t)}-1\right)^{2} p(t) d \mu+(\zeta-1)^{2}\right] \\
& \quad=\frac{1}{2}\left\|f^{\prime \prime}\right\|_{[r, R], \infty}\left[\int_{\Omega}\left(\frac{q^{2}(t)}{p(t)}-2 q(t)+p(t)\right) d \mu+(\zeta-1)^{2}\right] \\
& \quad=\frac{1}{2}\left\|f^{\prime \prime}\right\|_{[r, R], \infty}\left[\int_{\Omega} \frac{q^{2}(t)}{p(t)} d \mu-1+(\zeta-1)^{2}\right] \\
& \quad=\frac{1}{2}\left\|f^{\prime \prime}\right\|_{[r, R], \infty}\left[\int_{\Omega}\left(\frac{q^{2}(t)}{p(t)}-p(t)\right) d \mu+(\zeta-1)^{2}\right] \\
& \quad=\frac{1}{2}\left\|f^{\prime \prime}\right\|_{[r, R], \infty}\left[\int_{\Omega}\left(\frac{q^{2}(t)}{p^{2}(t)}-1\right) p(t) d \mu+(\zeta-1)^{2}\right] \\
& \quad=\frac{1}{2}\left\|f^{\prime \prime}\right\|_{[r, R], \infty}\left[D_{\chi^{2}}(p, q)+(\zeta-1)^{2}\right] ;
\end{aligned}
$$

and this completes the proof.

Proposition 7 Under the assumptions of Proposition 6, iff' is convex or $f_{ \pm}^{\prime \prime}$ exists, then we have

$$
\begin{aligned}
& \left|I_{f}(p, q)-f(\zeta)-(1-\zeta) f^{\prime}(\zeta)+\frac{f_{+}^{\prime \prime}(r)+f_{-}^{\prime \prime}(R)}{4}\left[D_{\chi^{2}}(p, q)+(\zeta-1)^{2}\right]\right| \\
& \quad \leq \frac{1}{4}\left|f_{-}^{\prime \prime}(R)-f_{+}^{\prime \prime}(r)\right|\left[D_{\chi^{2}}(p, q)+(\zeta-1)^{2}\right]
\end{aligned}
$$

for $\zeta \in[r, R]$. Some particular cases of interest are obtained by setting $\zeta=(r+R) / 2$ and $\zeta=1$.

Proof When $f^{\prime}$ is convex, we set $\gamma=f_{+}^{\prime \prime}(r)$ and $\Gamma=f_{-}^{\prime \prime}(R)(c f$. Remark 2). For the case where $f_{ \pm}^{\prime \prime}$ exists, we set $\gamma$ and $\Gamma$ appropriately to the values of $f_{+}^{\prime \prime}(r)$ and $f_{-}^{\prime \prime}(R)$, with $\gamma \leq \Gamma$. Utilising (3.3) for $g(t)=q(t) / p(t)$ and the measure $\int_{\Omega} p(t) d \mu=1$, we have

$$
\begin{aligned}
& \mid \int_{\Omega} f\left(\frac{q(t)}{p(t)}\right) p(t) d \mu-f(\zeta)-\left(\int_{\Omega} q(t) d \mu-\zeta\right) f^{\prime}(\zeta) \\
& \quad+\frac{f_{+}^{\prime \prime}(r)+f_{-}^{\prime \prime}(R)}{4} \int_{\Omega}\left(\frac{q(t)}{p(t)}-\zeta\right)^{2} p(t) d \mu \mid \\
& \quad=\left|I_{f}(p, q)-f(\zeta)-(1-\zeta) f^{\prime}(\zeta)+\frac{f_{+}^{\prime \prime}(r)+f_{-}^{\prime \prime}(R)}{4}\left[D_{\chi^{2}}(p, q)+(\zeta-1)^{2}\right]\right| \\
& \quad \leq \frac{1}{4}\left|f_{-}^{\prime \prime}(R)-f_{+}^{\prime \prime}(r)\right|\left[\int_{\Omega}\left(\frac{q(t)}{p(t)}-1\right)^{2} p(t) d \mu+(\zeta-1)^{2}\right] \\
& \quad=\frac{1}{4}\left|f_{-}^{\prime \prime}(R)-f_{+}^{\prime \prime}(r)\right|\left[D_{\chi^{2}}(p, q)+(\zeta-1)^{2}\right] .
\end{aligned}
$$


Note that we make use of the following:

$$
\begin{aligned}
\int_{\Omega}\left(\frac{q(t)}{p(t)}-\zeta\right)^{2} p(t) d \mu & =\int_{\Omega}\left(\frac{q(t)}{p(t)}\right)^{2} p(t) d \mu-1+(\zeta-1)^{2} \\
& =\int_{\Omega}\left(\left(\frac{q(t)}{p(t)}\right)^{2}-1\right) p(t) d \mu+(\zeta-1)^{2} \\
& =D_{\chi^{2}}(p, q)+(\zeta-1)^{2}
\end{aligned}
$$

and this completes the proof.

Example 1 If we consider the convex function $f:(0, \infty) \rightarrow \mathbb{R}, f(t)=t \log (t)$, then

$$
I_{f}(p, q)=\int_{\Omega} p(t) \frac{q(t)}{p(t)} \log \left(\frac{q(t)}{p(t)}\right) d \mu(t)=\int_{\Omega} q(t) \log \left(\frac{q(t)}{p(t)}\right) d \mu(t)=D_{K L}(q, p) .
$$

We have $f^{\prime}(t)=\log (t)+1$ and $f^{\prime \prime}(t)=1 / t$. By Proposition 6 , we have the following inequalities:

$$
\begin{aligned}
& \left|D_{K L}(q, p)-\zeta \log (\zeta)-(1-\zeta)(\log (\zeta)+1)\right| \\
& \quad=\left|D_{K L}(q, p)-1+\zeta-\log (\zeta)\right| \\
& \quad \leq \frac{1}{2}\left[\sup _{x \in[r, R]} \frac{1}{x}\right]\left[D_{\chi^{2}}(p, q)+(\zeta-1)^{2}\right] \\
& \quad=\frac{1}{2 r}\left[D_{\chi^{2}}(p, q)+(\zeta-1)^{2}\right]
\end{aligned}
$$

for all $\zeta \in[r, R]$; and when $\zeta=1$,

$$
0 \leq D_{K L}(q, p) \leq \frac{1}{2 r} D_{\chi^{2}}(p, q) .
$$

Furthermore, by Proposition 7, we have the inequalities:

$$
\begin{aligned}
& \left|D_{K L}(q, p)-\log (\zeta)-1+\zeta+\frac{r+R}{4 r R}\left[D_{\chi^{2}}(p, q)+(\zeta-1)^{2}\right]\right| \\
& \quad \leq \frac{R-r}{4 r R}\left[D_{\chi^{2}}(p, q)+(\zeta-1)^{2}\right]
\end{aligned}
$$

for $\zeta \in[r, R]$; and when $\zeta=1$,

$$
\left|D_{K L}(q, p)+\frac{r+R}{4 r R} D_{\chi^{2}}(p, q)\right| \leq \frac{R-r}{4 r R} D_{\chi^{2}}(p, q) .
$$

Example 2 If we consider the convex function $f:(0, \infty) \rightarrow \mathbb{R}, f(t)=-\log (t)$, then

$$
I_{f}(p, q)=-\int_{\Omega} p(t) \log \left(\frac{q(t)}{p(t)}\right) d \mu(t)=\int_{\Omega} p(t) \log \left(\frac{p(t)}{q(t)}\right) d \mu(t)=D_{K L}(p, q) .
$$

We have $f^{\prime}(t)=-1 / t$ and $f^{\prime \prime}(t)=1 / t^{2}$, and we note that

$$
\int_{\Omega} \frac{p^{2}(t)}{q(t)} d \mu=D_{\chi^{2}}(q, p)+1
$$


By Proposition 6, we have the following inequalities:

$$
\begin{aligned}
& \left|D_{K L}(p, q)+\log (\zeta)+\frac{1}{\zeta}-1\right| \\
& \quad \leq \frac{1}{2}\left[\sup _{x \in[r, R]} \frac{1}{t^{2}}\right]\left[D_{\chi^{2}}(p, q)+(\zeta-1)^{2}\right] \\
& \quad=\frac{1}{2 r^{2}}\left[D_{\chi^{2}}(p, q)+(\zeta-1)^{2}\right]
\end{aligned}
$$

for all $\zeta \in[r, R]$; and when $\zeta=1$,

$$
-\frac{1}{2 r^{2}} D_{\chi^{2}}(p, q) \leq 0 \leq D_{K L}(p, q) \leq \frac{1}{2 r^{2}} D_{\chi^{2}}(p, q) .
$$

Recall the following inequality from [12]:

$$
\left|D_{K L}(p, q)-D_{\chi^{2}}(q, p)\right| \leq \frac{1}{2 r^{2}} D_{\chi^{2}}(p, q),
$$

or equivalently,

$$
D_{\chi^{2}}(q, p)-\frac{1}{2 r^{2}} D_{\chi^{2}}(p, q) \leq D_{K L}(p, q) \leq D_{\chi^{2}}(q, p)+\frac{1}{2 r^{2}} D_{\chi^{2}}(p, q) .
$$

Thus, we have the following chain of inequalities:

$$
\begin{aligned}
-\frac{1}{2 r^{2}} D_{\chi^{2}}(p, q) & \leq D_{\chi^{2}}(q, p)-\frac{1}{2 r^{2}} D_{\chi^{2}}(p, q) \leq D_{K L}(p, q) \\
& \leq \frac{1}{2 r^{2}} D_{\chi^{2}}(p, q) \leq D_{\chi^{2}}(q, p)+\frac{1}{2 r^{2}} D_{\chi^{2}}(p, q)
\end{aligned}
$$

Furthermore, by Proposition 7, we have the inequalities:

$$
\begin{aligned}
& \left|D_{K L}(p, q)+\log (\zeta)+\frac{1}{\zeta}-1+\frac{r^{2}+R^{2}}{4 r^{2} R^{2}}\left[D_{\chi^{2}}(p, q)+(\zeta-1)^{2}\right]\right| \\
& \quad \leq \frac{R^{2}-r^{2}}{4 r^{2} R^{2}}\left[D_{\chi^{2}}(p, q)+(\zeta-1)^{2}\right]
\end{aligned}
$$

for $\zeta \in[r, R]$; and when $\zeta=1$,

$$
-\frac{R^{2}-r^{2}}{4 r^{2} R^{2}} \leq D_{K L}(p, q)+\frac{r^{2}+R^{2}}{4 r^{2} R^{2}}\left[D_{\chi^{2}}(p, q)\right] \leq \frac{R^{2}-r^{2}}{4 r^{2} R^{2}} D_{\chi^{2}}(p, q) .
$$

Recall the following inequality from [12]:

$$
\left|D_{K L}(p, q)-D_{\chi^{2}}(q, p)+\frac{r^{2}+R^{2}}{4 r^{2} R^{2}}\left[D_{\chi^{2}}(p, q)\right]\right| \leq \frac{R^{2}-r^{2}}{4 r^{2} R^{2}} D_{\chi^{2}}(p, q),
$$

or equivalently,

$$
\begin{aligned}
D_{\chi^{2}}(q, p)-\frac{R^{2}-r^{2}}{4 r^{2} R^{2}} D_{\chi^{2}}(p, q) & \leq D_{K L}(p, q)+\frac{r^{2}+R^{2}}{4 r^{2} R^{2}}\left[D_{\chi^{2}}(p, q)\right] \\
& \leq D_{\chi^{2}}(q, p)+\frac{R^{2}-r^{2}}{4 r^{2} R^{2}} D_{\chi^{2}}(p, q) .
\end{aligned}
$$


Thus, we have the following chain of inequalities:

$$
\begin{aligned}
-\frac{R^{2}-r^{2}}{4 r^{2} R^{2}} D_{\chi^{2}}(p, q) & \leq D_{\chi^{2}}(q, p)-\frac{R^{2}-r^{2}}{4 r^{2} R^{2}} D_{\chi^{2}}(p, q) \\
& \leq D_{K L}(p, q)+\frac{r^{2}+R^{2}}{4 r^{2} R^{2}}\left[D_{\chi^{2}}(p, q)\right] \\
& \leq \frac{R^{2}-r^{2}}{4 r^{2} R^{2}} D_{\chi^{2}}(p, q) \\
& \leq D_{\chi^{2}}(q, p)+\frac{R^{2}-r^{2}}{4 r^{2} R^{2}} D_{\chi^{2}}(p, q) .
\end{aligned}
$$

\section{Conclusions}

We study the magnitude of:

$$
\int_{\Omega} f \circ g d \mu-f(\zeta)-\left(\int_{\Omega} g d \mu-\zeta\right) f^{\prime}(\zeta)-\frac{1}{2} \lambda \int_{\Omega}(g-\zeta)^{2} d \mu, \quad \zeta \in[a, b]
$$

to provide new inequalities of Jensen-Ostrowski type. In Remark 3, we provide a generalised version of inequality (1.8) (cf. Proposition 3) in the measure-theoretic (and probabilistic) form.

We obtain an inequality which gives a bound to the discrepancy in the Jensen integral inequality:

$$
\left|\int_{\Omega}(f \circ g) d \mu-f\left(\int_{\Omega} g d \mu\right)\right| \leq \frac{1}{2}\left\|f^{\prime \prime}\right\|_{[a, b], \infty} \sigma^{2}(g)
$$

in Theorem 4. In Remark 4, we consider a special case of the above inequality and compare it to the results (inequalities (1.5) and (1.6)) by Costarelli and Spigler [2]. Our result gives a better upper bound than (1.5), but (1.6) still gives the better upper bound, due to the stronger assumption of $C^{2}$ smoothness. Nevertheless, our result holds in a more general setting (a measure-theoretic and probabilistic form).

We obtain inequalities with bounds involving the $p$-norms $(1 \leq p \leq \infty)$ in Theorem 1 , inequalities for functions with bounded second derivatives in Theorem 2 , and inequalities for convex second derivatives in Theorem 3, with different types of convexity. In Remark 5, we show that the assumption of convexity gives refinement to the inequality in Theorem 4. Finally, we apply these inequalities for $f$-divergence measure in information theory in Section 5.

\section{Competing interests}

The authors declare that they have no competing interests.

\section{Authors' contributions}

PC, SSD and EK contributed equally in all stages of writing the paper. All authors read and approved the final manuscript.

\section{Author details}

'Department of Mathematics and Statistics, La Trobe University, Melbourne (Bundoora), 3086, Australia. ${ }^{2}$ School of Engineering and Science, Victoria University, P.O. Box 14428, Melbourne, Victoria 8001, Australia. ${ }^{3}$ School of Computer Science and Applied Mathematics, University of the Witwatersrand, Private Bag 3, Wits, 2050, South Africa. ${ }^{4}$ Department of Pure and Applied Mathematics, University of Johannesburg, P.O. Box 524, Auckland Park, 2006, South Africa.

\section{Acknowledgements}

The research of E Kikianty is supported by the Claude Leon Foundation (South Africa). 


\section{References}

1. Pečarić, JE, Proschan, F, Tong, YL: Convex Functions, Partial Orderings and Statistical Applications. Mathematics in Science and Engineering, vol. 187. Academic Press, Boston (1992)

2. Costarelli, D, Spigler, R: How sharp is the Jensen inequality? J. Inequal. Appl. 2015, 69 (2015)

3. Dragomir, SS: A Grüss type inequality for isotonic linear functionals and applications. Demonstr. Math. 36(3), 551-562 (2003)

4. Dragomir, SS: Reverses of the Jensen inequality in terms of first derivative and applications. Acta Math. Vietnam. 38(3), 429-446 (2013)

5. Ostrowski, A: Über die Absolutabweichung einer differentierbaren Funktionen von ihren Integralmittelwert. Comment. Math. Helv. 10, 226-227 (1938)

6. Cerone, P, Dragomir, SS, Roumeliotis, J: An inequality of Ostrowski type for mappings whose second derivatives are bounded and applications. East Asian Math. J. 15(1), 1-9 (1999)

7. Mitrinović, DS, Pečarić, JE, Fink, AM: Inequalities for Functions and Their Integrals and Derivatives. Kluwer Academic, Dordrecht (1994)

8. Dragomir, SS, Rassias, TM (eds.): Ostrowski Type Inequalities and Applications in Numerical Integration. Kluwer Academic, Dordrecht (2002)

9. Dragomir, SS: Jensen and Ostrowski type inequalities for general Lebesque integral with applications. RGMIA Res. Rep. Collect. 17, Article 25 (2014)

10. Dragomir, SS: New Jensen and Ostrowski type inequalities for general Lebesgue integral with applications. RGMIA Res. Rep. Collect. 17, Article 27 (2014)

11. Dragomir, SS: General Lebesgue integral inequalities of Jensen and Ostrowski type for differentiable functions whose derivatives in absolute value are h-convex and applications. RGMIA Res. Rep. Collect. 17, Article 38 (2014)

12. Cerone, P, Dragomir, SS, Kikianty, E: Jensen-Ostrowski type inequalities and applications for $f$-divergence measures. Appl. Math. Comput. 266, 304-315 (2015)

13. Dragomir, SS: Integral inequalities of Jensen type for $\lambda$-convex functions. RGMIA Res. Rep. Collect. 17, Article 18 (2014)

14. Bhattacharyya, A: On a measure of divergence between two statistical populations defined by their probability distributions. Bull. Calcutta Math. Soc. 35, 99-109 (1943)

15. Hellinger, E: Neue Bergrüirdung du Theorie quadratisher Formerus von uneudlichvieleu Veränderlicher. J. Reine Angew. Math. 36, 210-271 (1909)

16. Jeffreys, $\mathrm{H}$ : An invariant form for the prior probability in estimating problems. Proc. R. Soc. Lond. A 186, 453-461 (1946)

17. Kapur, JN: A comparative assessment of various measures of directed divergence. Adv. Manag. Stud. 3, 1-16 (1984)

18. Taneja, IJ: Generalised information measures and their applications. http://www.mtm.ufsc.br/ taneja/bhtml/bhtml.html

19. Topsoe, F: Some inequalities for information divergence and related measures of discrimination. IEEE Trans. Inf. Theory 46(4), 1602-1609 (2000)

20. Kullback, S, Leibler, RA: On information and sufficiency. Ann. Math. Stat. 22, 79-86 (1951)

21. Csiszár, II: On topological properties of $f$-divergences. Studia Sci. Math. Hung. 2, 329-339 (1967)

22. Csiszár, II, Körner, J: Information Theory: Coding Theorem for Discrete Memoryless Systems. Academic Press, New York (1981)

23. Vajda, I: Theory of Statistical Inference and Information. Kluwer Academic, Dordrecht (1989)

\section{Submit your manuscript to a SpringerOpen ${ }^{\odot}$ journal and benefit from:}

- Convenient online submission

Rigorous peer review

- Immediate publication on acceptance

- Open access: articles freely available online

- High visibility within the field

- Retaining the copyright to your article 